Article

\title{
Online Signature Analysis for Characterizing Early Stage Alzheimer's Disease: A Feasibility Study
}

\author{
Zelong Wang ${ }^{1}$, Majd Abazid ${ }^{1}$, Nesma Houmani ${ }^{1,2, *}{ }^{\mathbb{C}}$, Sonia Garcia-Salicetti ${ }^{1,2, *}$ and \\ Anne-Sophie Rigaud ${ }^{3,4}$ \\ 1 Electronics and Physics Department, Telecom SudParis, Institut Polytechnique de Paris, 9 rue Charles \\ Fourier, 91011 Evry, France \\ 2 SAMOVAR, Telecom SudParis, CNRS, Institut Polytechnique de Paris, 9 rue Charles Fourier, \\ 91011 Evry, France \\ 3 AP-HP, Groupe Hospitalier Cochin Paris Centre, Hôpital Broca, Pôle Gérontologie, 75013 Paris, France \\ 4 L'unité de Recherche Universitaire EA 4468, Université Paris Descartes, 75006 Paris, France \\ * Correspondence: Nesma.Houmani@telecom-sudparis.eu (N.H.); Sonia.Garcia@telecom-sudparis.eu (S.G.-S.)
}

Received: 6 August 2019; Accepted: 23 September 2019; Published: 29 September 2019

check for updates

\begin{abstract}
We aimed to explore the online signature modality for characterizing early-stage Alzheimer's disease (AD). A few studies have explored this modality, whereas many on online handwriting have been published. We focused on the analysis of raw temporal functions acquired by the digitizer on signatures produced during a simulated check-filling task. Sample entropy was exploited to measure the information content in raw time sequences. We show that signatures of early-stage AD patients have lower information content than those of healthy persons, especially in the time sequences of pen pressure and pen altitude angle with respect to the tablet. The combination of entropy values on two signatures for each person was classified with two linear classifiers often used in the literature: support vector machine and linear discriminant analysis. The improvements in sensitivity and specificity were significant with respect to the a priori group probabilities in our population of AD patients and healthy subjects. We show that altitude angle, when combined with pen pressure, conveys crucial information on the wrist-hand-finger system during signature production for pathology detection.
\end{abstract}

Keywords: online signature analysis; sample entropy; Alzheimer's disease; classification

\section{Introduction}

Signatures have long been used for personal biometric authentication. This behavioral modality, widely used in our daily lives, evolves with age. The production of fine motor movements strongly progresses during childhood and for many years until the motor program ruling handwritten movements reaches maturity. At this stage, the signing gesture becomes the outcome of a highly automated ballistic process, involving multiple areas of the brain [1]. Alterations in this automated and unconscious process may reflect a change in health condition, such as a decline in cognitive or motor functions.

Few researchers have examined handwriting signatures for assessing the health state of individuals. Prior studies on handwriting focused on other handwriting tasks, such as loops, letters, and handwritten texts, for characterizing and detecting different pathologies, such as Parkinson's disease [2-9], Huntington's [9], Alzheimer's [9-16], and depression [10].

Alzheimer's disease (AD), from the perspective of handwritten signatures analysis, has rarely been studied. To the best of our knowledge, only one study, specifically on online handwritten signatures [17], proposed a machine learning approach to discriminate AD patients from healthy subjects. The authors exploited the sigma-lognormal model [18] for online signatures to extract global 
features of each signature instance, such as the number of lognormal densities, the maximum speed divided by the signing time, and the number of peaks in the graphing speed normalized by their duration, average, and standard deviations of some key parameters of the sigma-lognormal model. Three classifiers were compared: a support vector machine (SVM) with a linear kernel, and two decision trees: classification and regression trees (CART) and bagging CART. The best results were obtained with the bagging CART algorithm; an error rate of $3 \%$ was reached on a private database of 32 healthy and 29 pathological signatures.

In the present work, we studied the relationship between handwriting movements and early-stage Alzheimer's disease (AD) by analyzing online signatures captured in a real-life task: signing a check. We propose considering the raw temporal functions acquired by the digitizer and using the sample entropy (SE) measure to quantify the possible alterations in temporal functions induced by the disease in the early stages. Our hypothesis is that since signing is uniquely both an automated and an unconscious writing pattern, a cognitive and motor disorder during the early stage of disease would be detectable by alterations in the raw temporal functions. This analysis may provide insights for early AD diagnosis.

Our paper is organized as follows: In Section 2, we detail the database and its associated acquisition protocol. In Section 3, we review the SE measure and present the experiments and results. Section 4 states our conclusions and perspectives for future work.

\section{Materials and Data Acquisition Protocol}

Our private dataset was acquired at Broca Hospital in Paris within the framework of the ALWRITE project $[15,16]$, a French research project on the analysis of different handwriting tasks for AD assessment. All participants freely signed a consent form after receiving information about the study. The whole protocol contained, among many others, a real-life-inspired task that involves filling out a simulated check printed on a sheet of paper and fixed to a digitizing tablet.

Two simulated checks (pseudo-checks) were provided on two different sheets of paper and presented consecutively to each participant. Each person naturally filled out the whole information in the check by appending the amount in words and numeric form, the date, the place, and their signature. Participants filled out both pseudo-checks on a Wacom Intuos Pro Large tablet with an inking pen. The digitizing tablet sampled the pen trajectory at $125 \mathrm{~Hz}$ and captured five raw temporal functions: pen position $(x(t), y(t))$, pen pressure $p(t)$, and two pen inclination angles, azimuth $A z(t)$ and altitude $\operatorname{Alt}(t)$, as shown in Figure 1.

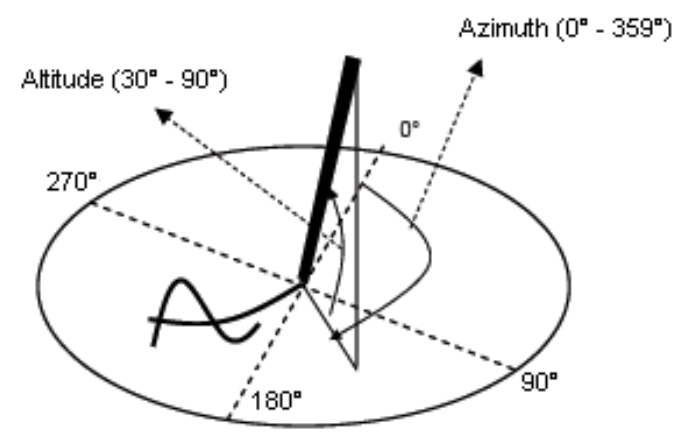

Figure 1. Azimuth and altitude angles captured by the Wacom digitizing tablet.

For our study, online signature data were manually segmented from the whole handwritten information written on the check (the amount in words, the amount in numeric form, the date, and the place). Examples of online signatures from one healthy subject and one early-stage AD patient are displayed in Figure 2. As the in-air trajectory of the pen (pen-up) is also captured by the digitizer up to $2 \mathrm{~cm}$ off the tablet surface, the signatures displayed in Figure 2 also show in-air trajectories in green points. 

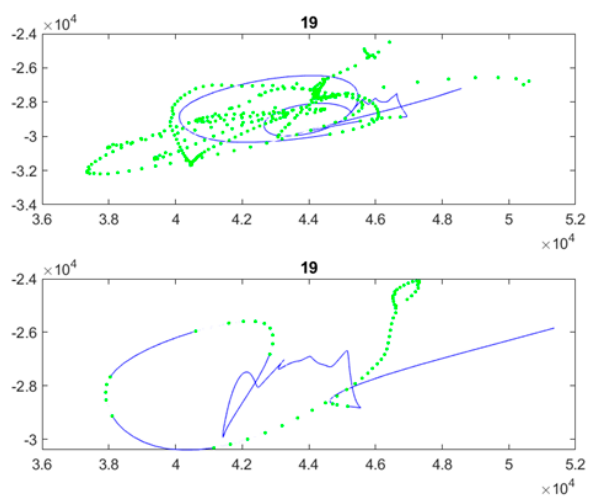

(a)
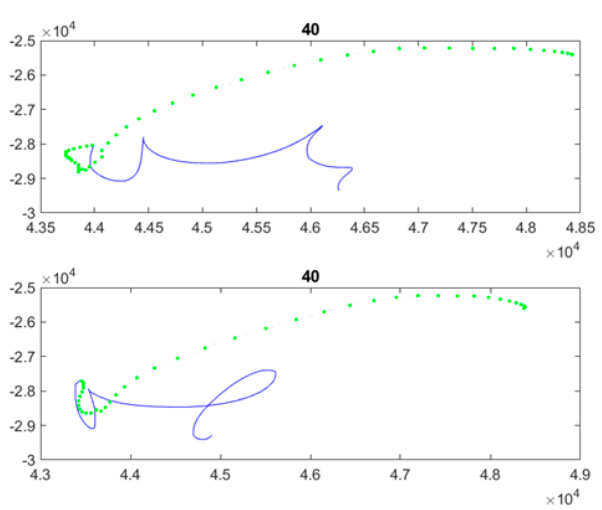

(b)

Figure 2. Examples of segmented signatures: (a) both signatures of an Alzheimer's disease (AD) patient; (b) both signatures of a healthy subject. Green points represent the in-air trajectory captured by the Wacom tablet.

Figures 3-7 display the five raw temporal functions for one AD signature, and a healthy one, belonging to the persons whose signatures are shown in Figure 2. We notice a different behavior between these two signatures in terms of their raw temporal functions.

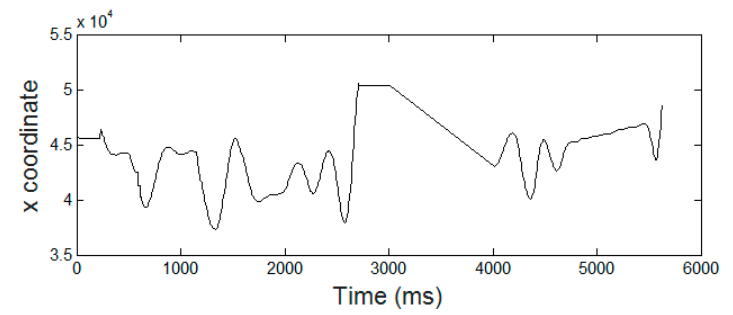

(a)

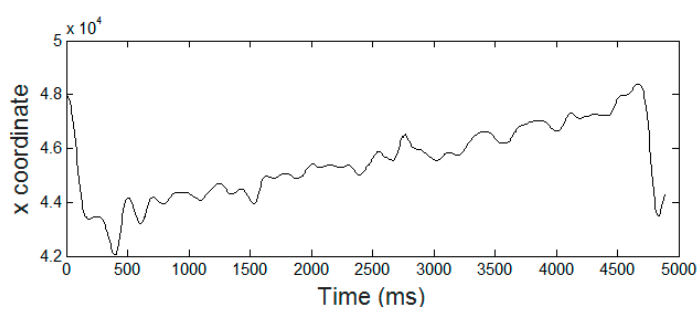

(b)

Figure 3. The $x$ coordinate temporal function for (a) an AD signature and (b) a healthy one.

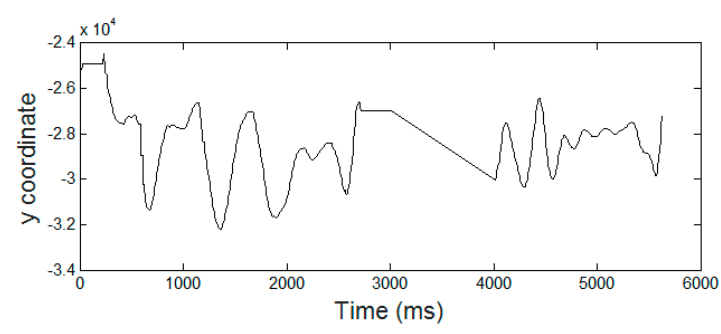

(a)

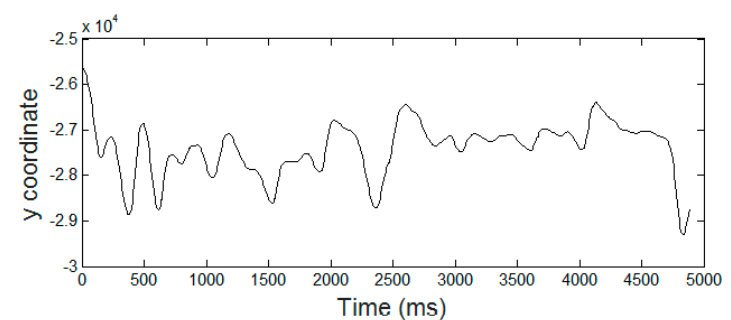

(b)

Figure 4. The $y$ coordinate temporal function for (a) an AD signature and (b) a healthy one.

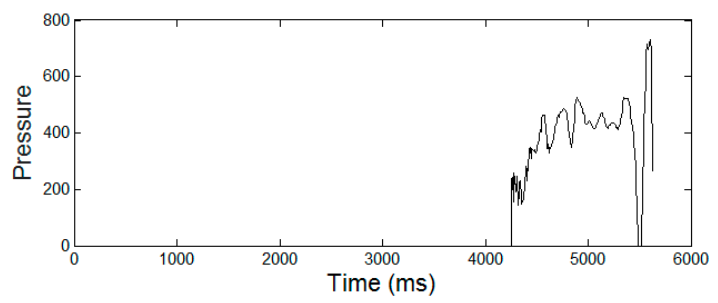

(a)

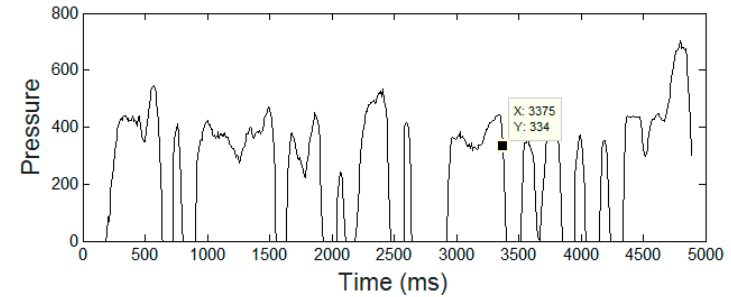

(b)

Figure 5. The pressure temporal function for (a) an AD signature and (b) a healthy one. 


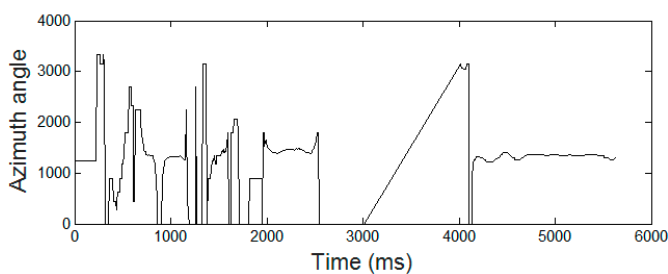

(a)

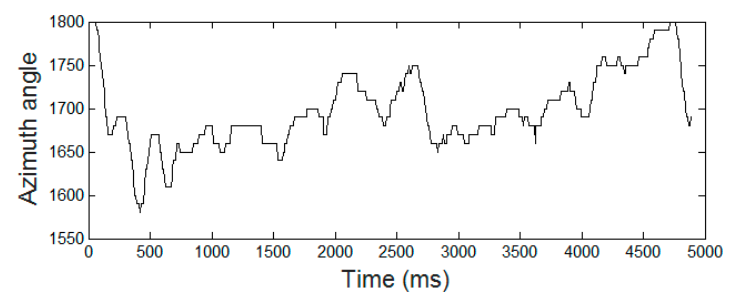

(b)

Figure 6. The azimuth angle for (a) an AD signature and (b) a healthy one.

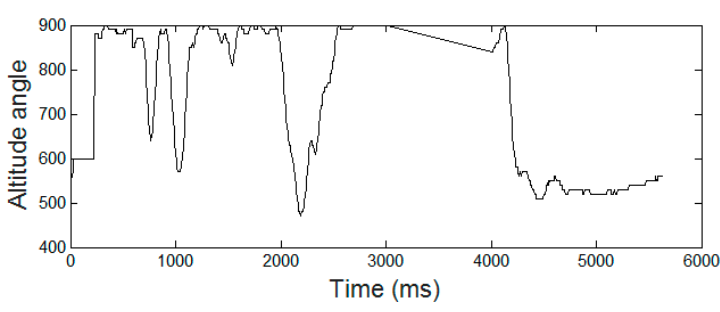

(a)

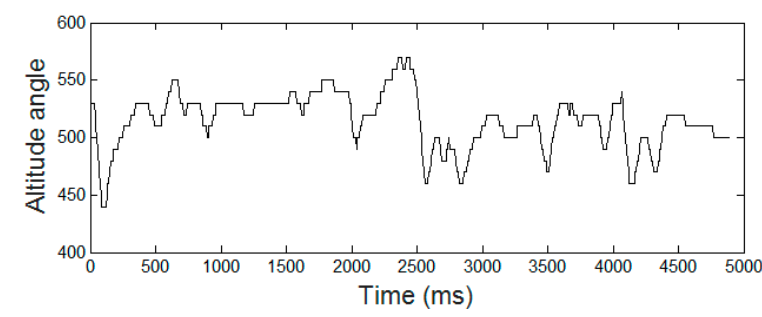

(b)

Figure 7. The altitude angle for (a) an AD signature and (b) a healthy one.

The used dataset contained online signatures of 70 participants. From the overall dataset, 31 participants were patients with early-stage $\mathrm{AD}$ (AD patients), and 39 were healthy controls (HC subjects). Table 1 summarizes the characteristics of the subjects. AD patients were diagnosed at Broca Hospital (Paris, France) based on the DSM-V criteria [19] and considered as having early-stage AD if their mini-mental state examination (MMSE) score was over 20 [19]. HC subjects performed neuropsychological tests to ensure their cognitive profile was normal. Participants with medical problems, such as stroke and other neurodegenerative diseases, were not included.

Table 1. Characteristics of the 70 subjects available in the dataset. HC denotes healthy controls, MMSE denotes mini-mental state examination.

\begin{tabular}{ccccccc}
\hline & \multicolumn{3}{c}{ Male (n= 21) } & \multicolumn{3}{c}{ Female (n = 49) } \\
\hline Number & $\begin{array}{c}\text { Age } \\
(\text { mean } \pm \text { std) }\end{array}$ & $\begin{array}{c}\text { MMSE } \\
(\text { mean } \pm \text { std) }\end{array}$ & Number & $\begin{array}{c}\text { Age } \\
\text { (mean } \pm \text { std) }\end{array}$ & $\begin{array}{c}\text { MMSE } \\
\text { (mean } \pm \text { std) }\end{array}$ \\
\hline $\begin{array}{c}\text { AD patients } \\
(\mathbf{n}=\mathbf{3 1})\end{array}$ & 14 & $81.5 \pm 7.4$ & $23.1 \pm 3.8$ & 17 & $78.2 \pm 6.6$ & $22.5 \pm 4.3$ \\
\hline $\begin{array}{c}\text { HC subjects } \\
(\mathbf{n = 3 9 )}\end{array}$ & 7 & $75.5 \pm 6.4$ & $28.8 \pm 0.7$ & 32 & $72.2 \pm 6.4$ & $28.8 \pm 1.1$ \\
\hline
\end{tabular}

\section{Methods and Analysis of Signatures}

\subsection{Sample Entropy Measure}

The concept of entropy has been widely and successfully exploited in the literature for the analysis of biomedical signals, especially for electroencephalography analysis [20,21]. More recently, SE was used on online handwritten inputs for health status assessment [22,23]. It is used to quantify irregularities in time sequences and measures complexity. To the best of our knowledge, SE has never been used for $\mathrm{AD}$ assessment based on signature analysis.

On-time series of length $N\{u(1), u(2), \ldots, u(i), \ldots, u(N)\}$, an integer value $m<N$ is fixed, specifying the length of the subsequences to be compared (window on the signal), and let $r$ be a positive real number. 
The following $(N-m+1)$ vectors $X_{m}(1), X_{m}(2), \ldots, X_{m}(i), \ldots, X_{m}(N-m+1)$ are defined as:

$$
X_{m}(i)=\{u(i), u(i+1), \ldots, u(i+m-1)
$$

and are embedded in the $m$-dimensional space, since each contains a subsequence of length $m$ starting at point $i$. The distance between two different vectors $(i \neq j)$ is defined as the maximum difference of their scalar components:

$$
d\left(X_{m}(i), X_{m}(j)\right)=\max _{0 \leq k \leq m-1}\{|u(i+k)-u(j+k)|\}
$$

SE is then:

$$
S E(m, r)=-\log (A / B)
$$

where $A$ is the number of template vectors pairs less than $r$ far in the embedded dimension $(m+1)$, and $B$ is the number of template vectors pairs less than $r$ far in the embedded dimension $m$. With construction $A \leq B$, we have $S E(m, r) \geq 0$.

In the literature [20-23], the hyper-parameters $m$ and $r$ are often fixed empirically with the objective to maximize the discrimination between the groups: between Parkinson patients and healthy subjects [22], between AD patients and healthy subjects [20,21], and between patients with essential tremor and healthy subjects [23]. In this work, we studied the variation in SE values for all possible values in a given interval for both hyper-parameters $m$ and $r$. The objective was to analyze the behavior of this measure independently of fixed values of its hyper-parameters.

\subsection{Initial Trends in the Correlation Between SE and Metadata}

We computed the SE values of all signatures for different values of $m=1, \ldots, 9$ and $r=0.1$ to 0.9 in 0.1 steps. The SE of a signature was computed considering the whole signing gesture, including both in-air and on-paper trajectories during the signing process.

To gain initial insights into its behavior, we computed the SE for each raw temporal function separately. Then, we analyzed the correlation between the obtained values and two metadata, the MMSE and age, and that for the 70 participants independently of the group (AD or HC).

The correlation values between the MMSE and the average SE on both signatures of each person for all values of $m$ and $r$ are reported in Table 2. We noticed a positive correlation between the MMSE and SE that was higher for the $y$ coordinate, pen pressure, and altitude angle compared with those computed for $x$ coordinate and azimuth angle. This result shows that persons with normal cognitive profiles (high MMSE) tend to produce signatures with more information content.

Table 2. Correlation values between the MMSE and sample entropy (SE) for the five temporal functions.

\begin{tabular}{ccccc}
\hline MMSE, SE $(x)$ & MMSE, SE $(y)$ & MMSE, SE $(p)$ & MMSE, SE(Alt) & MMSE, SE $(A z)$ \\
\hline 0.28 & 0.36 & 0.39 & 0.33 & 0.26 \\
\hline
\end{tabular}

Table 3 displays the correlation values between age and the average SE on both signatures for all values of $m$ and $r$. We noticed a negative correlation between age and SE for each temporal function. This reveals that, in general, with age, entropy tends to decrease, meaning that temporal functions show less intrinsic irregularities and variation in the signature gesture. The handwritten production loses information content with age.

Table 3. Correlation values between age and SE for the five temporal functions.

\begin{tabular}{ccccc}
\hline Age, SE $(x)$ & Age, SE(y) & Age, SE $(p)$ & Age, SE(Alt) & Age, SE(Az) \\
\hline-0.22 & -0.30 & -0.32 & -0.33 & -0.10 \\
\hline
\end{tabular}


We observed a very low correlation between age and azimuth compared with other temporal functions (-0.1). Aging appears to have no effect on this parameter. Azimuth could be used to distinguish a right-handed from a left-handed person, for example, for biometric authentication purposes. In our dataset, except one person, all the other participants were right-handed and thus had a similar azimuth profile. Therefore, our dataset had less variance in azimuth between persons compared with all other temporal functions. This is why we did not consider the azimuth temporal function in the following studies.

\subsection{Influence of Signature Styles}

SE quantifies the intrinsic information content in signatures, and thus, may be sensitive to signature style. For this reason, we addressed whether $\mathrm{HC}$ and $\mathrm{AD}$ patients produced different signature styles and studied the influence of style on SE.

Signatures of $\mathrm{HC}$ and $\mathrm{AD}$ patients were labeled visually by three experts into text-based signatures (where all the allographs are legible), mixed (where one or more but not all of the allographs are legible), and stylized (where none of the allographs are legible) [24]. These three styles may be related to signature complexity, quantified by SE. The resulting distribution in each style is reported in Table 4 .

Table 4. Distribution of signature styles in AD and HC groups.

\begin{tabular}{cccc}
\hline Group & Stylized & Mixed & Text-Based \\
\hline $\begin{array}{c}\text { AD } \\
(\mathbf{n}=31)\end{array}$ & 21 & 10 & 0 \\
\hline $\begin{array}{c}\text { HC } \\
(\mathbf{n}=39)\end{array}$ & 21 & 18 & 0 \\
\hline
\end{tabular}

We first noticed that text-based signatures do not exist in our dataset; no signatures were completely legible.

To study the influence of signature stylization, we considered the SE values computed for the $x$ coordinate (SE $(x))$ and $y$ coordinate $(\mathrm{SE}(y))$, and that for all signatures, independent of the group (AD or HC). Then, we performed $k$-means clustering, with $k=3$, on both entropy values simultaneously (two-dimensional (2D) clustering). Table 5 reports the distribution of signature styles in the obtained three groups and their respective bi-dimensional centroids.

Table 5. Distribution of stylized and mixed signature styles in the three resulting groups.

\begin{tabular}{cccc}
\hline & Group 1 & Group 2 & Group 3 \\
\hline [mean SE $(\boldsymbol{x})$, mean SE $(y)]$ & {$[0.2,0.34]$} & {$[0.14,0.23]$} & {$[0.11,0.16]$} \\
Stylized signatures $(\mathbf{n}=\mathbf{4 2})$ & 2 & 30 & 10 \\
Mixed signatures $(\mathbf{n}=\mathbf{2 8})$ & 0 & 24 & 4 \\
\hline
\end{tabular}

We observed that $77.14 \%$ of persons with stylized and mixed signatures were grouped together in Group 2 ( 54 of the 70 participants). This means that most signatures with such styles have similar information content in the $x(t)$ and $y(t)$ temporal functions. Also, two persons with stylized signatures had the highest SE values, which means that an illegible signature could be complex.

These results indicate that SE quantifies irregularities in temporal functions independent of the stylization in the form. Given these findings, we then studied the relationship between SE and AD independent of signature styles.

\subsection{Relationship between $S E$ and $A D$}

In each group (AD vs. HC), we analyzed the impact of the hyper-parameters $m$ and $r$ on SE values computed for the four temporal functions $(x(t), y(t), p(t)$, and Alt $(t))$. In the surfaces displayed in both 
Figures 8 and 9, we noticed that the SE measure was sensitive to the hyper-parameter $r$ and not to $m$. Also, for low values of $r$, SE values were much higher for HC compared with AD patients. This means that the intrinsic variation in the temporal functions in the signature was much stronger for healthy persons compared with AD patients. We, thus observed a significant information loss in Alzheimer's signatures for low values of hyper-parameter $r$ in Figure 8 (for $x(t)$ and $y(t)$ pen coordinates) and Figure 9 (for pen pressure $p(t)$ and altitude angle $\operatorname{Alt}(t)$ ).

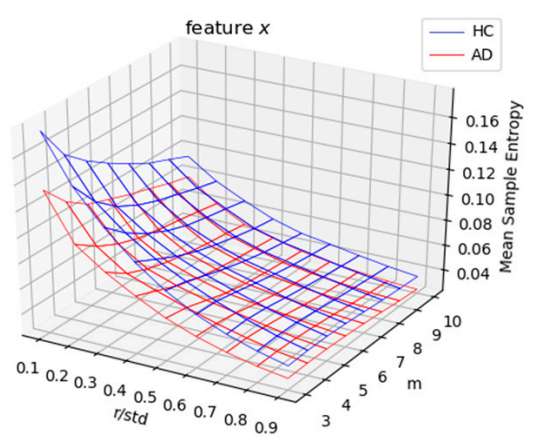

(a)

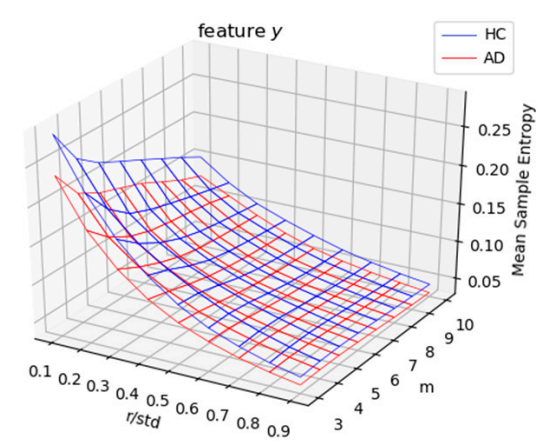

(b)

Figure 8. Average SE values on AD patients (red) vs. HC subjects (blue) for different values of the hyper-parameters $m$ and $r$ ( $r$ divided by the standard deviation (std) of the corresponding raw signal) considering the (a) $x(t)$ and (b) $y(t)$ coordinates.

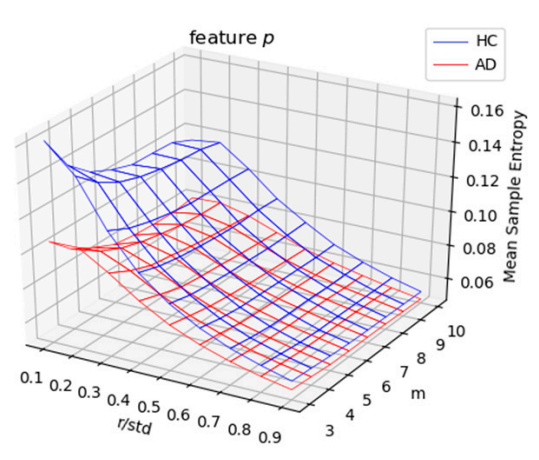

(a)

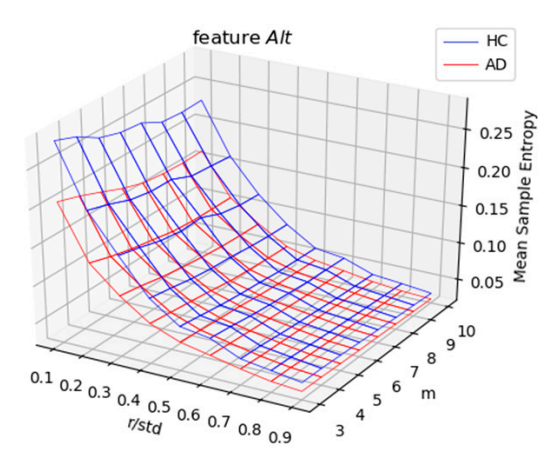

(b)

Figure 9. Average SE values on $\mathrm{AD}$ patients (red) vs. HC subjects (blue) for different values of the hyper-parameters $m$ and $r$ ( $r$ divided by the standard deviation (std) of the corresponding raw signal) considering: (a) pen pressure $p(t)$ and (b) pen altitude angle Alt $(t)$.

To gain further insight into the impact of the two hyper-parameters on SE, Figures 10-13 display boxplots of the entropy values for the four temporal functions when fixing one hyper-parameter and varying the other. These figures show that SE is more sensitive to $r$ compared with $m$. We observed that when $r$ increases, entropy values decrease for both $\mathrm{AD}$ and HC groups and their variances for persons in each group decrease. When $m$ increases, the entropy values and their variances for persons in each group tend to remain stable. 


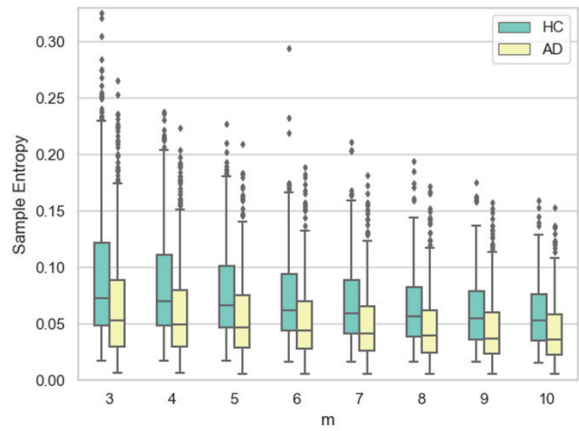

(a)

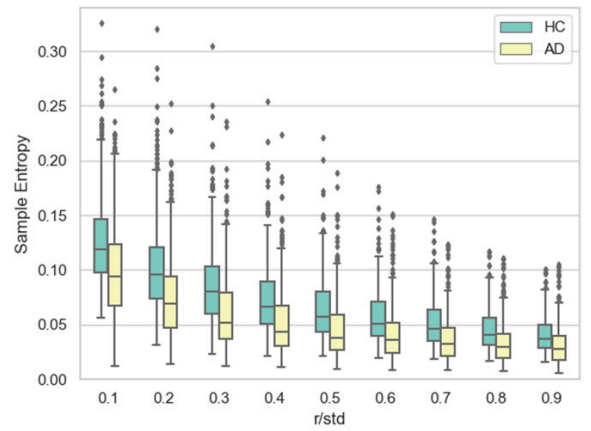

(b)

Figure 10. Boxplots of SE values for $\mathrm{AD}$ and HC considering $x(t)$ coordinate for (a) each value of $m$ when varying $r$ and (b) each value of $r$ when varying $m$.

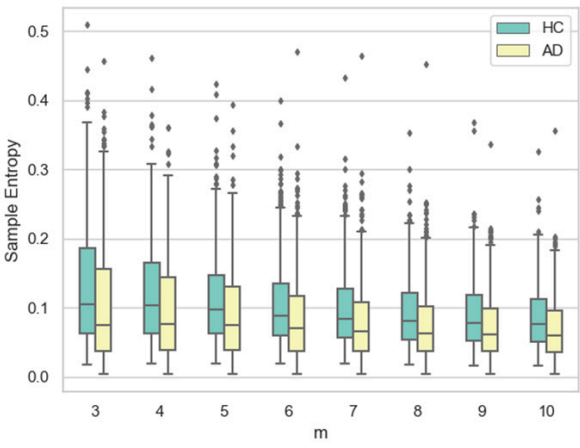

(a)

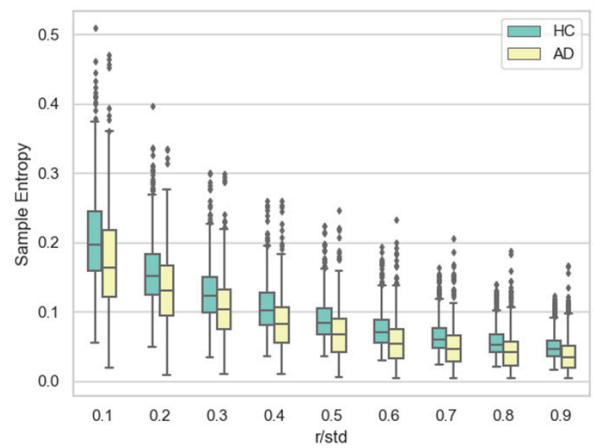

(b)

Figure 11. Boxplots of SE values for AD and HC considering the $y(t)$ coordinate for (a) each value of $m$ when varying $r$ and (b) each value of $r$ when varying $m$.

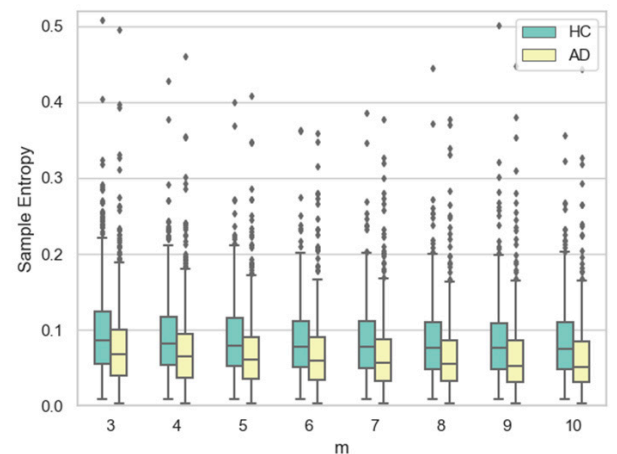

(a)

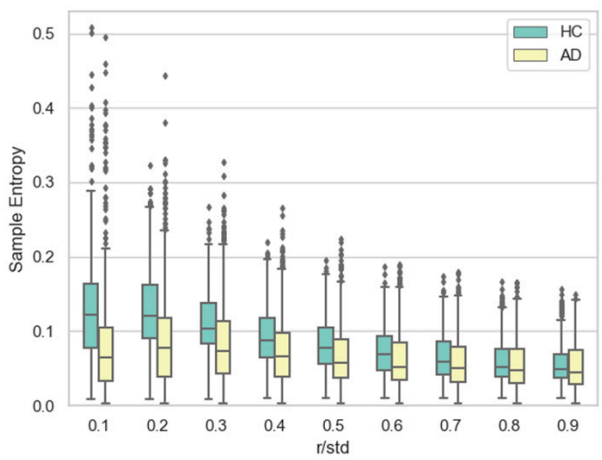

(b)

Figure 12. Boxplots of SE values for AD and HC considering pen pressure $p(t)$ for (a) each value of $m$ when varying $r$ and (b) each value of $r$ when varying $m$.

We also noticed that entropy values are much higher for temporal function $y(t)$ compared with $x(t)$, indicating that signatures vary more vertically than horizontally. The same occurs for altitude: it had higher information content than horizontal movement $(x(t))$. The altitude angle conveys information about how the writer holds and moves the pen when signing, and is particularly modified by the intensity of vertical movement $(y(t))$. Therefore, strong variations in $y(t)$ also appear in the temporal function $A l t(t)$. 


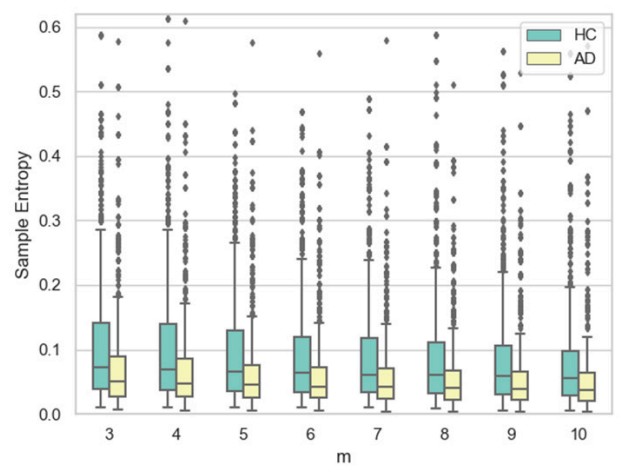

(a)

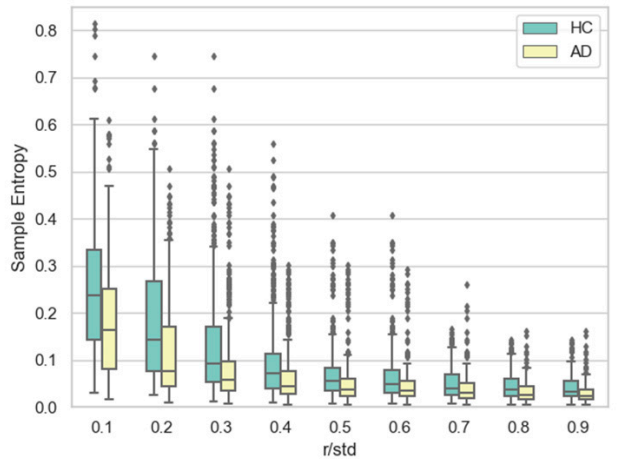

(b)

Figure 13. Boxplots of $\mathrm{SE}$ values for $\mathrm{AD}$ and $\mathrm{HC}$ considering pen altitude angle $A l t(t)$ for (a) each value of $m$ when varying $r$ and(b) each value of $r$ when varying $m$.

Concerning information loss in Alzheimer's signatures relative to healthy subjects, observed for low values of $r$ in Figures 8 and 9, the phenomenon was clearly stronger for pen pressure and pen altitude angle, particularly for $m=3$ and $r / s t d=0.1$. For pen pressure and pen altitude, we found a relative increase of entropy of around $50 \%$ and $37 \%$ between signatures of AD patients and those of healthy subjects, respectively.

\subsection{Towards AD Detection: AD vs. HC Classification Results}

To study the discriminative power of SE between AD and HC subjects, as SE is sensitive to $r$, we performed classification for $m=3$ and $r / s t d=0.1$. For these values of hyper-parameters, the Mann-Whitney test in Table 6 shows a significant difference between the entropy values of AD and $\mathrm{HC}$ populations for the four temporal functions.

Table 6. The $p$-values computed with the Mann-Whitney test for the four temporal functions.

\begin{tabular}{ccccc}
\hline Mann-Whitney Test & $x(t)$ & $y(t)$ & $p(t)$ & Alt $(t)$ \\
\hline$p$-value & $6 \times 10^{-5}$ & 0.0013 & $3.12 \times 10^{-6}$ & $4.22 \times 10^{-4}$ \\
\hline
\end{tabular}

Based on this result, we considered two linear classifiers, a linear support vector machine (SVM) and linear discriminant analysis (LDA), using a two-fold cross-validation protocol for training and testing. For each raw temporal function considered, the SE values of both signatures for each person were used as inputs to the classifier.

Tables 7 and 8 report the obtained sensitivity (percentage of AD patients correctly classified), specificity (percentage of HC correctly classified), and accuracy values for the SVM and LDA classifiers.

Table 7. Classification results for the support vector machine (SVM) classifier with two-fold cross-validation.

\begin{tabular}{ccccc}
\hline Classification & $p(t)$ & $A l t(t)$ & $p(t), A l t(t)$ & $x(t), y(t), p(t), A l t(t)$ \\
\hline Sensitivity & $58.06 \%$ & $61.29 \%$ & $64.52 \%$ & $74.19 \%$ \\
Specificity & $76.92 \%$ & $79.49 \%$ & $71.79 \%$ & $76.92 \%$ \\
Accuracy & $68.80 \%$ & $71.43 \%$ & $68.57 \%$ & $75.71 \%$ \\
\hline
\end{tabular}


Table 8. Classification results for the linear discriminant analysis (LDA) classifier with two-fold cross-validation.

\begin{tabular}{ccccc}
\hline Classification & $p(t)$ & Alt $(t)$ & $p(t), \operatorname{Alt}(\boldsymbol{t})$ & $x(t), y(t), p(t), \boldsymbol{A l t}(\boldsymbol{t})$ \\
\hline Sensitivity & $54.84 \%$ & $54.84 \%$ & $64.52 \%$ & $67.74 \%$ \\
Specificity & $79.49 \%$ & $71.79 \%$ & $79.49 \%$ & $58.97 \%$ \\
Accuracy & $68.57 \%$ & $64.28 \%$ & $72.86 \%$ & $62.85 \%$ \\
\hline
\end{tabular}

We noticed that for both SVM and LDA classifiers, the combination of SE values computed for pen pressure and pen altitude improved the sensitivity relative to taking each of those features separately. As the a priori group probability for the dataset here used was 0.44 for the AD group (31 AD patients out of 70) and 0.56 for the HC group (39 subjects out of 70), the improvement obtained was significant. For sensitivity, which was our main objective, the relative improvement was $45.7 \%$ with both classifiers ( $64.52 \%$ in sensitivity relative to $44.28 \%$ of a priori Alzheimer's group probability).

When combining the SE values of the four temporal functions, the sensitivity (up to $74.19 \%$ ) and specificity (up to $76.92 \%$ ) improved significantly for the SVM classifier. For the LDA classifier, sensitivity also improved (up to $67.74 \%$ ) but the specificity decreased by almost $26 \%$.

Thus, in the best-case scenario, with the SVM and the combination of the four SE values, the relative improvements in sensitivity and specificity were $67.54 \%$ and $37.35 \%$ relative to the a priori group probability of correct classification of $\mathrm{AD}$ patients and $\mathrm{HC}$ subjects, respectively.

\section{Discussion and Conclusions}

In this work, we analyzed the sample entropy (SE) of raw temporal signals captured by a digitizer on signatures appended during a simulated check-filling task. Our extensive study of the effect of the hyper-parameters when measuring SE added to a statistical analysis highlighted that online signatures written by early-stage AD patients show significantly lower entropy values, and thus, lower information content, than those of healthy persons. We verified that SE is not influenced by different signature styles, only represented in our dataset by mixed and stylized signatures [24].

We observed that the difference in the information content between signatures of AD patients and those of $\mathrm{HC}$ was high for pen pressure and altitude angle time sequences. This result that the pen altitude angle allows characterizing the pathology with pen pressure on signatures is a novel finding. Notably, in former work on biometrics, pen inclination angles were found to be misleading for characterizing a writer (with or without pressure) [25]. Our analysis instead showed that AD patients have less tonus in their way of holding the pen, which impacts pen pressure and variations in the vertical direction, which are lower, compared with those of healthy subjects. Our classification results suggest that altitude, when combined with pen pressure, conveys valuable information about the wrist-hand-finger system during signature production for pathology detection. In other words, one of our findings is that the way the pen is held by the writer during the signing gesture has significance for AD detection. Our statistical analysis with the Mann-Whitney test showed that a significant difference exists between $\mathrm{AD}$ and $\mathrm{HC}$ distributions of $\mathrm{SE}$ values for pen coordinates, pressure, and altitude angle. When combining these four temporal functions, we obtained an accuracy of $75.71 \%$ with a linear SVM classifier in the framework of a two-fold cross-validation protocol to ensure the reliability of the classification results.

Finally, the experiments and analyses conducted to support our claim—quantifying the alterations in raw temporal functions with SE, describing the complete signing gesture in the air and on paper, provides insight into early AD diagnosis. Our study shows that online signature, despite being a signal of short duration, conveys valuable information about a writer's motor and cognitive skills through its ballistic, automated, and unconscious process, and is thus a possible modality for the automatic health state assessment.

However, our study has some limitations: (1) our dataset is small, both in terms of the number of persons in each group (AD and $\mathrm{HC}$ ), and the number of signatures per person; (2) we exploited 
only the raw temporal functions captured by the digitizer for the data description; and (3) even if we showed that a statistically significant difference exists between $\mathrm{AD}$ and HC populations with the SE measure, we obtained a misclassification rate of around $24 \%$ with a linear SVM classifier. The rather low correlation coefficients between SE and the MMSE suggest that SE is not sufficient alone to finely characterize the alterations in temporal functions induced by the disease.

In the future, we will address these limitations by (1) incorporating other online signatures captured during another task in the ALWRITE project; (2) considering other kinematic parameters such as speed, acceleration, and jerk, successfully used for AD assessment based on handwriting after a local point-wise feature extraction [15,16]; and (3) combining SE with other non-linear measures to propose a more refined detection of alterations in signatures due to the disease.

We also aim to extend this transversal study to signatures acquired in different sessions. The longitudinal framework will contribute to evaluating the viability of our approach for quantifying the degradations in signatures according to dementia severity.

Author Contributions: Conceptualization-N.H.; Data curation-Z.W. and M.A.; Formal analysis-M.A., N.H. and S.G.-S.; Investigation-Z.W., M.A., and N.H.; Methodology-N.H. and S.G.-S.; Resources-S.G.-S. and A.-S.R.; Software-Z.W., M.A., and N.H.; Supervision-N.H. and S.G.-S.; Validation-N.H. and S.G.-S.; Visualization-Z.W., N.H., and S.G.-S.; Writing-original draft-N.H. and S.G.-S.

Funding: This research was funded by Institut Mines-Telecom (France) and MAIF Foundation (France).

Acknowledgments: We would like to thank Hospital Broca (Paris, France) for enabling the data acquisition campaign, and especially Christian Kahindo and Victoria Cristancho-Lacroix, who largely contributed to the acquisition of data in the framework of the French ALWRITE research project.

Conflicts of Interest: The authors declare no conflict of interest.

\section{References}

1. Caligiuri, M.P.; Linton, A.M. The Neuroscience of Handwriting, 1st ed.; CRC Press, Taylor \& Francis Group: Boca Raton, FL, USA, 2012; pp. 35-55.

2. Teulings, H.L.; Stelmach, G.E. Control of stroke size, peak acceleration, and stroke duration in Parkinsonian handwriting. Hum. Mov. Sci. 1991, 10, 315-334. [CrossRef]

3. Teulings, H.L.; Contreras-Vidal, J.L.; Stelmach, G.E.; Adler, C.H. Parkinsonism reduces coordination of fingers, wrist, and arm in fine motor control. Exp. Neurol. 1997, 146, 159-170. [CrossRef]

4. Drotar, P.; Mekyska, J.; Rektorova, I.; Masarova, L.; Smekal, Z.; Faundez-Zanuy, M. A new modality for quantitative evaluation of Parkinson's disease: In-air movement. In Proceedings of the IEEE 13th International Conference Bioinformatics Bioengineering, Chania, Greece, 10-13 November 2013; pp. 1-4.

5. Rosenblum, S.; Samuel, M.; Zlotnik, S.; Erikh, I.; Schlesinger, I. Handwriting as an objective tool for Parkinson's disease diagnosis. J. Neurol. 2013, 260, 2357-2361. [CrossRef] [PubMed]

6. Thomas, M.; Lenka, A.; Kumar Pal, P. Handwriting Analysis in Parkinson Disease: Current Status and Future Directions. Mov. Disord. Clin. Pract. 2017, 4, 806-818. [CrossRef] [PubMed]

7. Moetesum, M.; Siddiqi, I.; Vincent, N.; Cloppet, F. Assessing visual attributes of handwriting for prediction of neurological disorders-A case study on Parkinson's disease. Pattern Recognit. Lett. 2018, 121, 19-27. [CrossRef]

8. Senatore, R.; Della Cioppa, A.; Marcelli, A. Automatic Diagnosis of Neurodegenerative Diseases: An Evolutionary Approach for Facing the Interpretability Problem. Information 2019, 10, 30. [CrossRef]

9. Slavin, M.J.; Phillips, J.L.; Bradshaw, K.; Hall, K.A.; Presnell, I. Consistency of handwriting movements in dementia of the Alzheimer's type: A comparison with Huntington's and Parkinson's diseases. J. Int. Neuropsychol. Soc. 1999, 5, 20-25. [CrossRef] [PubMed]

10. Schröter, A.; Mergl, R.; Bürger, K.; Hampel, H.; Möller, H.J.; Hegerl, U. Kinematic analysis of handwriting movements in patients with Alzheimer's disease, Mild Cognitive Impairment, Depression and healthy subjects. Dement. Geriatr. Cognit. Disord. 2003, 15, 132-142. [CrossRef] [PubMed]

11. Werner, P.; Rosenblum, S.; Bar-On, G.; Heinik, J.; Korczyn, A. Handwriting process variables discriminating mild Alzheimer's disease and Mild Cognitive Impairment. J. Gerontol. Psychol. Sci. 2006, 61, 228-236. [CrossRef] [PubMed] 
12. Yan, J.H.; Rountree, S.; Massman, P.; Smith Doody, R.; Li, H. Alzheimer's disease and Mild Cognitive Impairment deteriorate fine movement control. J. Psychiatr. Res. 2008, 42, 1203-1212. [CrossRef] [PubMed]

13. Garre-Olmo, J.; Faundez-Zanuy, M.; Lopez-de-Ipina, K.; Calvo-Perxas, L.; Turro-Garriga, O. Kinematic and pressure features of handwriting and drawing: Preliminary results between patients with Mild Cognitive Impairment, Alzheimer disease and healthy controls. Curr. Alzheimer Res. 2017, 14, 960-968. [CrossRef] [PubMed]

14. Ghaderyan, P.; Abbasi, A.; Saber, S. A new algorithm for kinematic analysis of handwriting data; towards a reliable handwriting-based tool for early detection of Alzheimer's disease. Expert Syst. Appl. 2018, 114, 428-440. [CrossRef]

15. El-Yacoubi, M.; Garcia-Salicetti, S.; Kahindo, C.; Rigaud, A.S.; Cristancho-Lacroix, V. From Aging to Early-Stage Alzheimer's: Uncovering Handwriting Multimodal Behaviors by Semi-Supervised Learning and Sequential Representation Learning. Pattern Recognit. 2019, 86, 112-133. [CrossRef]

16. Kahindo, C.; El-Yacoubi, M.; Garcia-Salicetti, S.; Rigaud, A.-S.; Cristancho-Lacroix, V. Characterizing Early Stage Alzheimer through Spatiotemporal Dynamics of Handwriting. IEEE Signal Process. Lett. 2018, 25, 1136-1140. [CrossRef]

17. Pirlo, G.; Diaz, M.; Ferrer, M.A.; Impedovo, D. Early Diagnosis of Neurodegenerative Diseases by Handwritten Signature Analysis. In Proceedings of the 2015 International Conference on Image Analysis and Processing, ICIAP 2015 Workshops, LNCS 9281, Las Vegas, NV, USA, 27-30 July 2015; pp. 290-297.

18. Dijoua, M.; Plamondon, R. Studying the variability of handwriting patterns using the kinematic theory. Hum. Mov. Sci. 2009, 28, 588-601. [CrossRef] [PubMed]

19. American Psychiatric Association. Diagnostic and Statistical Manual of Mental Disorders, 5th ed.; American Psychiatric Association: Arlington, VA, USA, 2013.

20. Abasolo, D.; Hornero, R.; Espino, P.; Alvarez, D.; Poza, J. Entropy analysis of the EEG backgroundactivity in Alzheimer's disease patients. Physiol. Meas. 2006, 27, 241-253. [CrossRef] [PubMed]

21. Houmani, N.; Dreyfus, G.; Vialatte, F. Epoch-based entropy for early screening of Alzheimer's disease. Int. J. Neural Syst. 2015, 25, 1-18. [CrossRef] [PubMed]

22. Ünlü, A.; Brause, R.; Krakow, K. Handwriting Analysis for Diagnosis and Prognosis of Parkinson's Disease. In Proceedings of the 2006 International Symposium on Biological and Medical Data Analysis, Thessalonik, Greece, 7-8 December 2006; Maglaveras, N., Chouvarda, I., Koutkias, V., Brause, R., Eds.; Springer: Heidelberg, Germany, 2006; Volume 4345, pp. 441-450.

23. Lopez de Ipina, K.; Solé-Casals, J.; Faundez-Zanuy, M.; Calvo, P.; Sesa, E.; Martinez de Lizarduy, U.; Bergareche, A. Selection of Entropy based Features for Automatic Analysis of Essential Tremor. Entropy 2016, 18, 184. [CrossRef]

24. Mohammed, L.A.; Found, B.; Caligiuri, M.; Rogers, D. The dynamic character of disguise behavior for text-based, mixed, and stylized signatures. J. Forensic Sci. 2011, 56 (Suppl. S1), S136-S141. [CrossRef]

25. Houmani, N.; Garcia-Salicetti, S.; Dorizzi, B. On assessing the robustness of Pen Coordinates, Pen Pressure and Pen Inclination to Short-term and Long-term Time Variability with Personal Entropy. In Proceedings of the IEEE Third International Conference on Biometrics: Theory, Applications and Systems (BTAS), Washington, DC, USA, 28-30 September 2009; pp. 1-6.

(C) 2019 by the authors. Licensee MDPI, Basel, Switzerland. This article is an open access article distributed under the terms and conditions of the Creative Commons Attribution (CC BY) license (http://creativecommons.org/licenses/by/4.0/). 